\title{
Wearable high-performance pressure sensors based on three-dimensional electrospun conductive nanofibers
}

\author{
O. Young Kweon', Sang Jin Lee ${ }^{1}$ and Joon Hak Oh (1)
}

\begin{abstract}
Polymer-based pressure sensors play a key role in realizing lightweight and inexpensive wearable devices for healthcare and environmental monitoring systems. Here, conductive core/shell polymer nanofibers composed of poly (vinylidene fluoride-co-hexafluoropropene) (PVDF-HFP)/poly(3,4-ethylenedioxythiophene) (PEDOT) are fabricated using three-dimensional (3D) electrospinning and vapor deposition polymerization methods, and the resulting sponge-like 3D membranes are used to create piezoresistive-type pressure sensors. Interestingly, the PEDOT shell consists of well-dispersed spherical bumps, leading to the formation of a hierarchical conductive surface that enhances the sensitivity to external pressure. The sponge-like 3D mats exhibit a much higher pressure sensitivity than the conventional electrospun 2D mats due to their enhanced porosity and pressure-tunable contact area. Furthermore, large-area, wireless, $16 \times 10$ multiarray pressure sensors for the spatiotemporal mapping of multiple pressure points and wearable bands for monitoring blood pressure have been fabricated from these 3D mats. To the best of our knowledge, this is the first report of the fabrication of electrospun 3D membranes with nanoscopically engineered fibers that can detect changes in external pressure with high sensitivity. The developed method opens a new route to the mass production of polymer-based pressure sensors with high mechanical durability, which creates additional possibilities for the development of human-machine interfaces.
\end{abstract}

\section{Introduction}

Flexible, sensitive, lightweight, and inexpensive pressure sensors are well suited for applications in portable and wearable devices. Wireless sensing devices enable users to monitor and record their physiological data in real time $^{1-3}$. Recently, artificial electronic skin (E-skin) that can simultaneously detect subtle pressure changes, the humidity, and the temperature has been intensively studied for its potential use in various applications, including health monitoring systems, medical diagnostics, soft robotics, and human-machine interfaces ${ }^{4-7}$. It has been demonstrated that flexible electronic devices and systems can be integrated with the human body or E-skin in the form of implantable, stick-on, or wearable electronics ${ }^{8,9}$. Particularly, integrated wearable pressure sensors have been developed with several types of transduction systems, including resistive, capacitive, and piezoelectric types ${ }^{10}$. Although manufacturing a sensing matrix on a pixel-by-pixel basis is common for pressure sensors, a sensor array may also be manufactured by embedding a pressure-sensitive layer into electrode-array platforms. Three-dimensional (3D) structured conductive polymeric materials are promising for achieving highly flexible pressure sensors. In the last few years, various structures and materials have been developed to fabricate ultrasensitive pressure sensors that can effectively collect pressure stimuli in the low-pressure regime $(<10 \mathrm{kPa})^{11-16}$. However, several obstacles limit the practical application of such sensors. Flexible pressure sensors often require elaborate nanostructured designs, and thus their

\footnotetext{
Correspondence: Joon Hak Oh (joonhoh@postech.ac.kr)

${ }^{1}$ Department of Chemical Engineering, Pohang University of Science and

Technology (POSTECH), Pohang, Gyeongbuk 37673, Korea
} 
fabrication requires multiple steps, high-vacuum conditions, and time-consuming syntheses ${ }^{17-21}$.

Electrospinning has been widely employed to overcome these limitations and can be used in the fabrication of nanofibers (NFs) or microfibers composed of numerous materials, such as elastic polymers, conductive materials, and graphen $\mathrm{e}^{22-25}$. In addition, electrospinning is suitable for rapidly producing large-area membranes with adjustable properties (high surface area, pore volume, and material type) under ambient conditions ${ }^{20,23}$. The most common method of fabricating highly conductive NFs is the direct electrospinning of conductive composite polymers on a flexible substrate. However, the formulation of composite polymers required to prepare an electrospinnable solution often degrades the intrinsic electrical properties of the conductive species. Additionally, dipping or immersing electrospun fibers in a solution containing a conductive material requires multiple steps to produce conductive fibers, and the irregular surface degrades the performance of the device ${ }^{26,27}$. The electrospinning procedure with a dual nozzle also needs sophisticated finetuned processes to obtain continuous uniform fibers ${ }^{28}$. In addition, these conventional electrospun structures typically form two-dimensional (2D) networks with fibers aligned parallel to the collector ${ }^{22,29}$. Layer-by-layer fibrous structures made by conventional 2D electrospinning tend to form with less film porosity in the perpendicular direction than in the planar direction. This phenomenon has limited the enhancement of 3D porosity in electrospun materials.

Piezoresistive sensors are a typical pressure sensor that convert applied pressure to a resistance signal and have been widely used because they are simple, low cost, and easy to operate. Conductive polymer films or elastomeric rubbers, typically filled with conductive carbon black particles, are used as sensing materials for piezoresistive sensors ${ }^{30}$. However, these conductive polymer thin film or rubber-based sensors are relatively insensitive, unstable, and difficult to reproduce in low-pressure regimes, which limit their practical applications. The piezoresistive sensing mechanism of conductive nanomaterials is based on a change in resistance resulting from variations in the contact area between conductors upon compressive deformation. The relatively low porosity of piezoresistive sensors made with conventional electrospun fibers results in small changes in resistance with changes in the ambient pressure and, consequently low-performance pressure sensors. The dense packing of NFs limits interchain movement and restricts changes in the resistance of the nanofibrillar membrane ${ }^{26}$. Recently, novel hybrid systems have been prepared using fabrication methods for creating various $3 \mathrm{D}$ structures $^{31,32}$, and the resulting systems boast a combination of high electronic conductivity ${ }^{33,34}$ and mechanical flexibility ${ }^{35,36}$. Despite the development of these new systems, a large demand for highly effective functional nanomaterials for use in high-performance flexible pressure sensors remains unmet.

In this study, we report the fabrication of highly conductive 3D electrospun poly(vinylidene fluorideco-hexafluoropropene) (PVDF-HFP)/poly(3,4-ethylenedioxythiophene) (PEDOT) mats with increased pore volume to enhance the pressure sensitivity and investigated their mechanoelectrical properties for applications in piezoresistive-type pressure sensors. We fabricated PVDF-HFP/PEDOT core/shell NFs with well-dispersed spherical conductive bumps using 3D electrospinning and vapor deposition polymerization (VDP $)^{37}$ methods for the core and shell components, respectively. Our method for producing core/shell NFs has a great advantage because the precursor solution enables the simultaneous building of a 3D structure and the initiation of polymerization, which also enables the formation of conductive spherical bumps. The sponge-like 3D membranes of these NFs exhibited bendable, elastic, and perfectly plastic properties and were used as pressure-sensitive layers for polymerbased piezoresistive-type pressure sensors. The fabricated 3D membranes exhibited a greatly enhanced sensitivity $\left(13.5 \mathrm{kPa}^{-1}\right)$ compared with those prepared using a conventional $2 \mathrm{D}$ electrospinning method $\left(5.1 \mathrm{kPa}^{-1}\right)$. The fabricated polymeric pressure-sensitive layers exhibited a minimum detection of $\sim 1 \mathrm{~Pa}$ and stable operation over 10,000 strain cycles. This high sensitivity and outstanding durability originated from the greatly enhanced porosity, pressure-tunable contact area, and embossing surface structure with spherical bumps. Furthermore, we created a durable 3D conductive structure and used it as a singlelayer sponge-like conductive pressure-sensitive material for a sensor array that can sense pressure pixel-by-pixel without modification of the existing electronic circuits, which simplified the manufacturing processes. The largearea, wireless, $16 \times 10$ multiarray pressure sensors for the spatiotemporal mapping of multiple pressure points and wearable wrist bands for monitoring blood pressure were successfully fabricated from these 3D mats, which were linked to a smartphone application for portable use. To the best of our knowledge, this is the first demonstration of the fabrication of an electrospun mat with randomly oriented 3D fibrous structures and its application in highly sensitive pressure sensors for monitoring environmental stimuli and health conditions.

\section{Materials and methods}

Preparation of 3D and conventional PVDF-HFP/PEDOT NFs PVDF-HFP/PEDOT NFs were fabricated using electrospinning and VDP methods. PVDF-HFP (M.W. 50,000-150,000 $\mathrm{g} \mathrm{mol}^{-1}$; Sigma-Aldrich, USA) and ferric chloride $\left(\mathrm{FeCl}_{3}, 97 \%\right.$; Sigma-Aldrich, USA) were mixed in a 1:1 dimethylformamide (DMF):tetrahydrofuran (THF) 
(w/w) solution (total concentration of solution, $18 \mathrm{wt} \%$ ). The solution was stirred at $60^{\circ} \mathrm{C}$ for $24 \mathrm{~h}$. The electrospinning equipment for fabricating PVDF-HFP NFs was purchased from Nano NC Corp. (Korea). Electrospinning of a single NF was performed at a voltage of $10.5 \mathrm{kV}$ using a single-tip electrospinning system (inner diameter of the nozzle, $0.33 \mathrm{~mm}$ ). The tip was connected to the positive terminal of a power supply. The tip-to-collector distance was $3-12 \mathrm{~cm}$, and the feeding rate of the solution was $6-8$ $\mu \mathrm{L} \min ^{-1}$. The collector was an aluminum plate for the 3D mats and an insulating Lucite plate for the 2D mats. PVDF-HFP fibers were then electrospun directly onto inkjet-printed PEDOT:PSS/PET (vide infra) or indium tin oxide (ITO)-coated polyethylene naphthalate (PEN) substrates. The sponge-like PVDF-HFP mats were placed in a vacuum chamber for the VDP of 3,4-ethylenedioxythiophene (EDOT). Liquid EDOT monomer $(0.3 \mathrm{~g})$ was loaded into the reactor by injection. The monomer was injected at room temperature and completely vaporized by heating the reactor at $70^{\circ} \mathrm{C}$ for $5 \mathrm{~h}$. Polymerization of EDOT onto the surface of the PVDF-HFP NFs was performed by chemical oxidization with $\mathrm{FeCl}_{3}$. Finally, the NFs were uniformly coated with PVDF-HFP/PEDOT by VDP. Two separate panels composed of PVDF-HFP/ PEDOT/substrate and substrate were prepared and stacked in a tandem structure to prepare a pressure sensor matrix.

\section{Preparation of PEDOT:PSS electrodes}

A PEDOT:PSS solution was inkjet-printed with a $60-\mu \mathrm{m}$ pitch nozzle onto a PET substrate to form flexible electrodes. Computer-aided design of PEDOT:PSS electrodes was used to print electrodes using an electrohydrodynamic inkjet printer, which was purchased from ENJET Corp. (Korea). Each electrode consisted of five printed lines to fill up one whole electrode. After printing PEDOT:PSS electrodes on a $25-\mu \mathrm{m}$-thick PET substrate, an annealing process was performed at $65^{\circ} \mathrm{C}$ for $25 \mathrm{~min}$. Then, ethylene glycol was immediately introduced to remove residual PSS and enhance the conductivity, and the printed electrode sheets were washed with ethanol and dried for $2 \mathrm{~h}$.

\section{Characterization of PVDF-HFP/PEDOT NFs}

The morphologies of the electrospun PVDF-HFP/ PEDOT NFs were studied using a focused ion beam (FIB) scanning electron microscope (SEM) (Helios S600; Thermo Fisher Scientific, USA), an FE-SEM (S4800; Hitachi, Japan), and an HR-TEM (JEM-2100F; JEOL, Japan). In the sample preparation, nanomaterials were directly deposited onto a copper grid. The molecular structure of the fibers was characterized by FT-IR (VERTEX 70; Bruker, USA) in transmission mode.

\section{Electrical measurements}

The sheet resistances of the PVDF-HFP/PEDOT NFs were measured using a four-point probe method at room temperature. A Keithley 4200 semiconductor parameter analyzer (US) was used under ambient atmosphere for electrical characterization and the sensing tests. The current-voltage $(I-V)$ curves, response times, and detection ranges of the pressure sensors were measured with a Keithley 4200 analyzer with Cu electrodes attached to each layer of the fiber mat. The applied voltage was $1-10 \mathrm{~V}$. To examine the performance of each pressure sensor, a $z$-axis stage and a force gauge (Mark-10) were used to apply loads to the sensor pad and record the applied pressure.

\section{Internet of things (IoT) platform}

A large-area sensing IC board (Snowboard) was purchased from Kitronyx Corp. (Korea). The Snowboard is an Arduino Leonardo-compatible board with integrated force and touch-sensing controllers. The force-sensing IC and Snowboard software were developed by Kitronyx and can be connected to any resistive matrix sensor. The singleunit pressure sensor was designed by Kong Tech Corp. (Korea). The custom-made algorithm and program were accessible via Bluetooth. Copper tape and wire were used for the connections between the IC board and the sensors. The PEDOT:PSS/PET film and heavily doped silicon wafers ( $n$-type, $<0.004 \Omega \mathrm{cm}$ ) were used as substrates for the large-area sensor and the unit sensor, respectively.

\section{Results and discussion}

\section{Fabrication of 3D PVDF-HFP/PEDOT NFs}

The overall experimental procedure for the fabrication of PVDF-HFP/PEDOT devices is illustrated in Fig. 1a. The basic idea in the design of this polymeric material is to use a polar, stretchable polymer combined with a mobile, high ionic strength salt. Thereby, ion-dipole interactions can occur between the polar groups on the polymer and the ionic $\mathrm{Fe}$ salt. To make an electrospinnable polymer solution, we chose PVDF-HFP as the polar polymer network. First, a PVDF-HFP pellet and $\mathrm{FeCl}_{3}$ were dissolved in a solution of DMF and THF (1:1 by weight). $\mathrm{FeCl}_{3}$ was used as an ion $\left(\mathrm{Fe}^{3+}\right)$ source for building 3D nanostructures and as an oxidizing agent for the polymerization of EDOT (Fig. 1b). Electrospinning under high voltage produced a fluffy stack of fibers with a 3D configuration composed of elongated PVDF-HFP fibers. It is generally thought that the differences between the fibrillar structures produced by conventional 2D and 3D electrospinning methods originate from changes in the transient electrical forces when the fibers hit the collecting plate. A collecting plate with a high surface conductivity and/or a polymer system with a low electrical resistivity are essential for the formation of 3D scaffolds. 


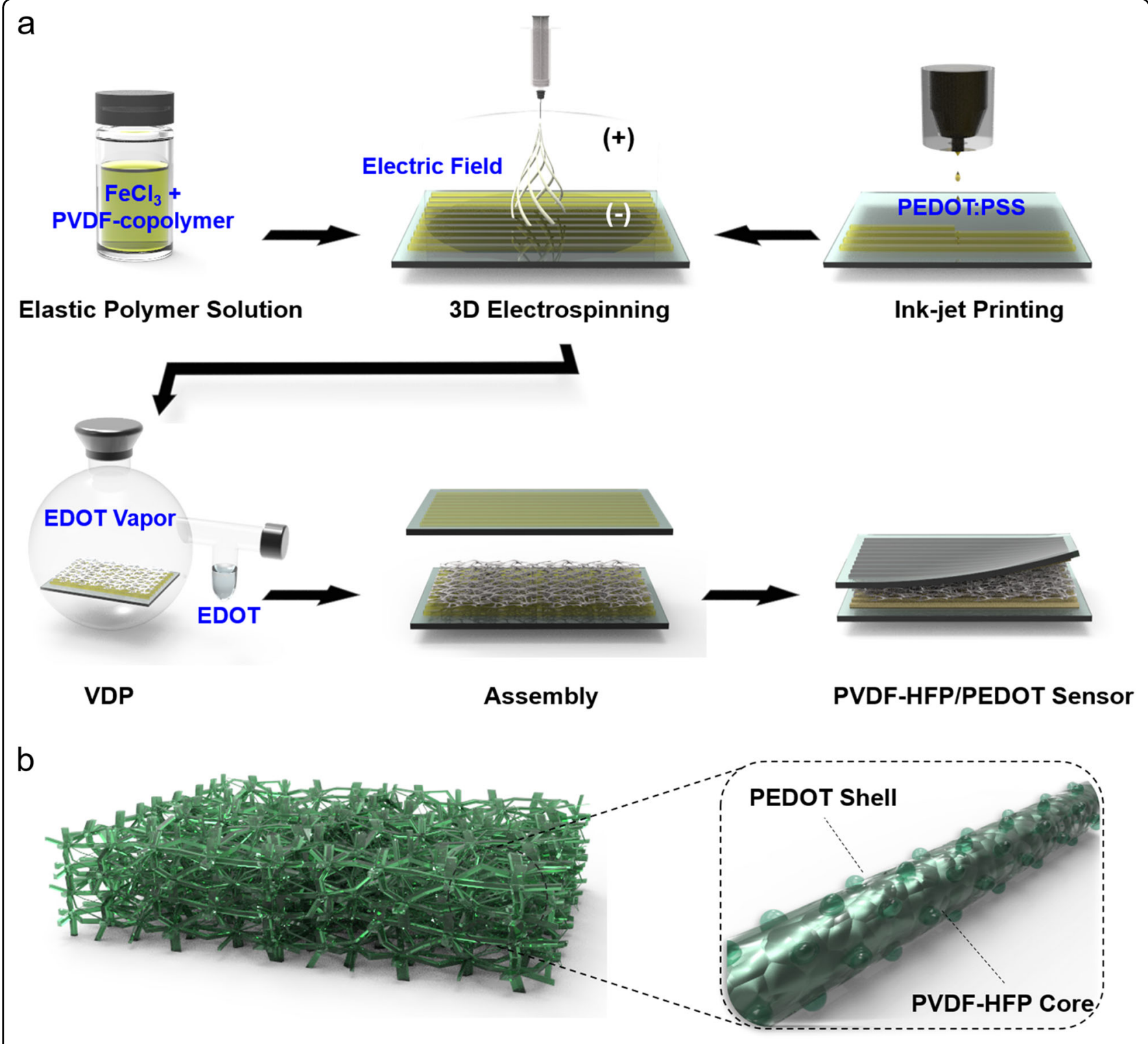

Fig. 1 Experimental set-up and a schematic image of PVDF-HFP/PEDOT mat. Schematic illustration of a the fabrication process of PVDF-HFP/ PEDOT NF-based piezoresistive pressure sensors and $\mathbf{b}$ electrospun 3D nanostructured PVDF-HFP/PEDOT NF mats

In a typical 3D electrospinning process, liquid droplets acquire negative charges and are elongated into fibers. The electrospun fibers with a highly negative surface charge move perpendicularly toward the collecting plate. When the fibers strike the metal collector, the static surface charge are rapidly transferred to the plate. Fewer electrons are left on the fibers, resulting in a decrease in the attractive interactions between the fibers and the collector. In some cases, single fibers can carry opposite charges in different regions depending on their proximity to the collector plate. The regions of a fiber close to the collector can carry positive charges and be repulsed by the collector, while the fiber ends remain negatively charged and are attracted to the collector ${ }^{38}$. Consequently, fibers are collected onto the plate in multiple orientations, resulting in loose and fluffy 3D scaffolds (Supplementary Fig. S1).

The resulting 3D random fiber network was spongelike, and the fibers were bendable and elastic. The vaporized EDOT monomers reacted with $\mathrm{Fe}^{3+}$ cations and polymerized via chemical oxidative polymerization on the surface of the PVDF-HFP fibers. The morphology and diameter of the as-prepared composite NFs were examined by high-resolution transmission electron microscopy (HR-TEM) as functions of the oxidant concentration and reaction time. The stepwise formation of a PEDOT shell 


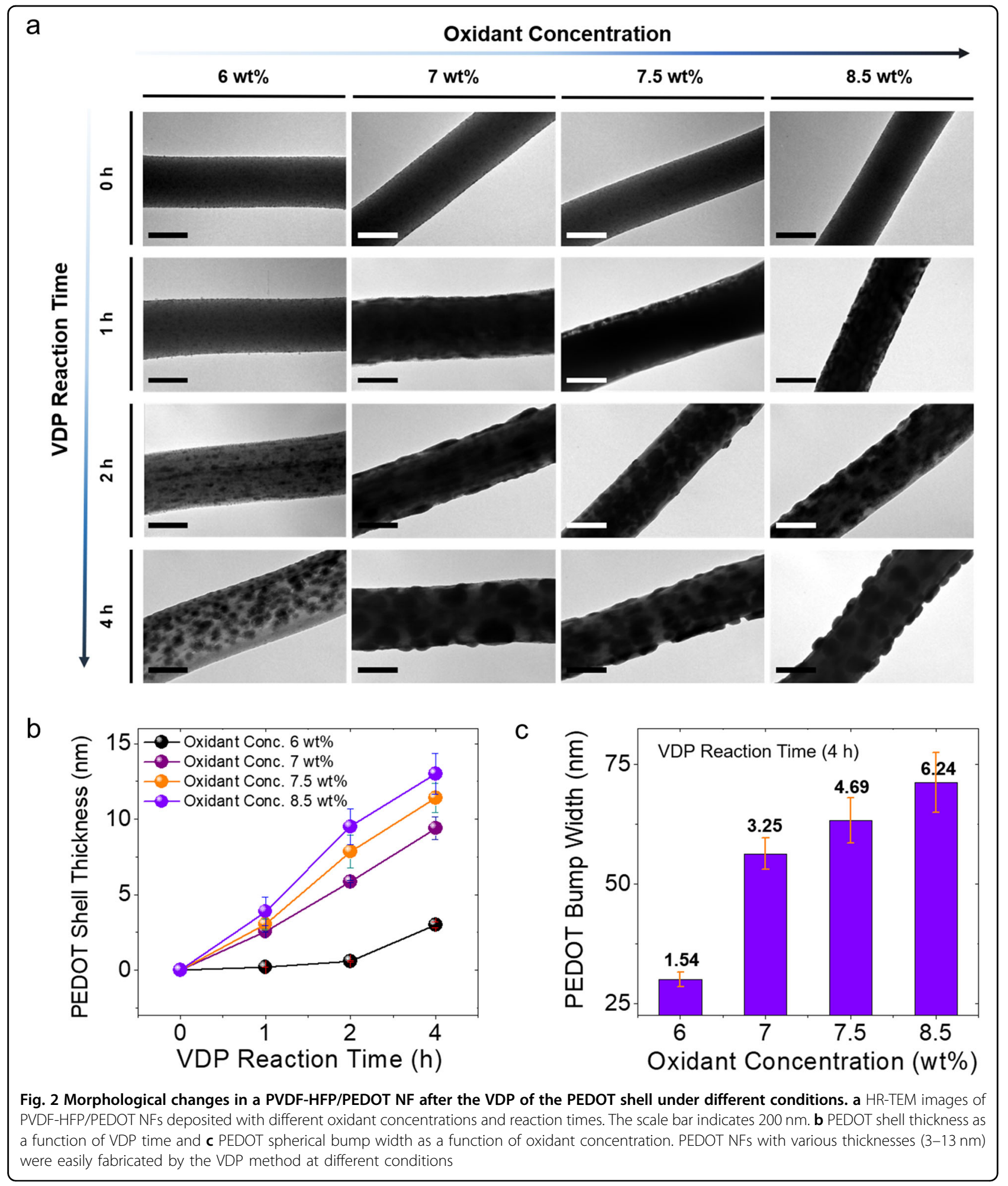

and spherical bumps on the surface of a PVDF-HFP fiber were clearly observed, as shown in Fig. 2a and Supplementary Fig. S2. The distance between NFs decreased gradually with increasing shell (PEDOT) thickness, which would eventually result in aggregation of the NFs during the process of VDP over a critical shell thickness, leading to an optimized shell thickness of ca. $13 \mathrm{~nm}$. The prepared core/shell NFs were directly deposited on a $\mathrm{Cu}$ grid, and the oxidant concentration and VDP reaction time were controlled. Finally, NFs covered with spherical PEDOT 

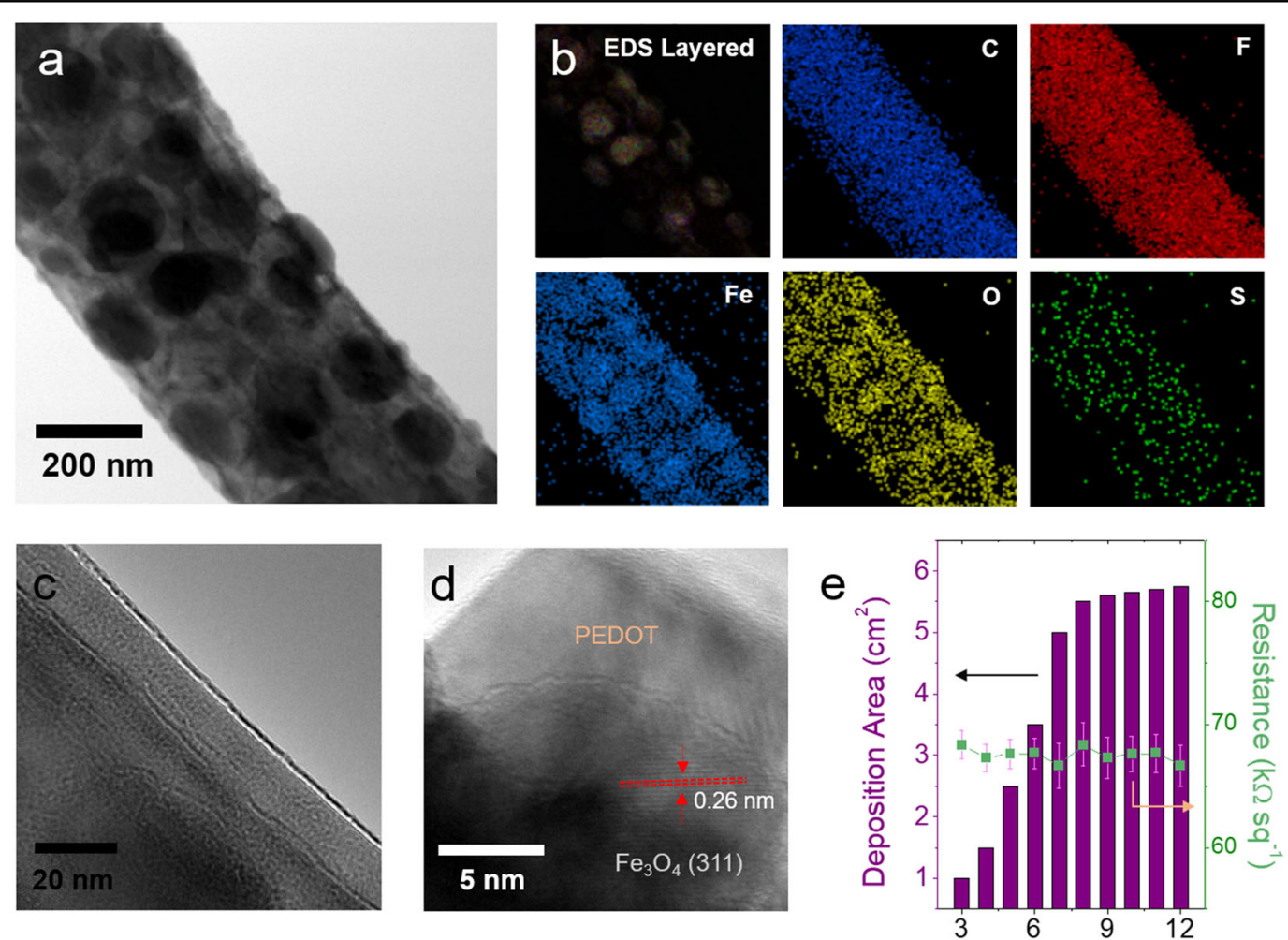

Tip-to-Collector Distance $(\mathrm{cm})$

Fig. 3 Structural analysis of a spherical bump PVDF-HFP/PEDOT NF. a HR-TEM image of a PVDF-HFP/PEDOT NF and $\mathbf{b}$ energy-dispersive spectrometer (EDS) mapping images of $\mathrm{C}, \mathrm{F}, \mathrm{Fe}, \mathrm{O}$, and $\mathrm{S}$ for the composite nanofibers. Enlarged HR-TEM images of $\mathbf{c}$ the shell and $\mathbf{d}$ a spherical bump of a PVDF-HFP/PEDOT NF. e Tip-to-collector distance vs. the deposition area of the collected NF mat from the 3D electrospinning process and the sheet resistance distribution of a PVDF-HFP/PEDOT NF mat deposited for 1 min

bumps with various thicknesses $(3-13 \mathrm{~nm})$ were successfully fabricated by the VDP method (Fig. 2b, c).

As shown in Fig. 3a, the average diameter of an optimized PVDF-HFP/PEDOT fiber was $480 \pm 34 \mathrm{~nm}$. The spherical PEDOT bumps also formed in a hierarchical manner. The successful fabrication of PVDF-HFP/PEDOT fibers was also confirmed by energy-dispersive spectroscopy (EDS) and Fourier transform infrared spectroscopy (FT-IR). The EDS data show that the Fe ions contained in the electrospinning solution were dispersed uniformly, creating dot-shaped islands throughout the NF after electrospinning. Elemental mapping analyses revealed that carbon $(61.59 \%)$, fluoride (25.52\%), oxygen $(11.20 \%)$, sulfur $(0.13 \%)$, and iron $(1.56 \%)$ were spread over the surface of the NFs (Fig. 3b, Supplementary Fig. S3 and Table S1). As a result, Fe cations adsorbed to the surface of the PVDF-HFP NFs due to charge-charge interactions between the $\mathrm{Fe}^{3+}$ ions and $\mathrm{F}^{-}$groups of PVDF-HFP. When the Fe cations were sufficiently spread on the fiber surface, coaxial PVDF-HFP/PEDOT NFs were prepared.

A uniform PEDOT shell was formed by vapor polymerization; the shell thickness was ca. $13 \mathrm{~nm}$, as shown in Fig. 3c. Under ambient conditions, $\mathrm{Fe}^{3+}$ ions were oxidized to $\mathrm{Fe}_{3} \mathrm{O}_{4}$ with a lattice fringe spacing of $0.26 \mathrm{~nm}$, as evidenced by the HR-TEM micrograph in Fig. 3d; this spacing is ascribed to the (311) plane of $\mathrm{Fe}_{3} \mathrm{O}_{4}$. Residual $\mathrm{Fe}^{3+}$ ions acted as oxidants that allowed the vaporized EDOT to polymerize on the surface of the $\mathrm{Fe}_{3} \mathrm{O}_{4}$-decorated fibers. In addition, spherical PEDOT bumps formed on the well-dispersed $\mathrm{Fe}_{3} \mathrm{O}_{4}$ islands, resulting in hierarchically structured conductive fibers (Fig. 3d). Typical absorption bands corresponding to the $\mathrm{C}-\mathrm{H}$ stretching of thiophene rings and in-plane $\mathrm{C}-\mathrm{H}$ stretching were observed at 1183 and $1211 \mathrm{~cm}^{-1}$, respectively (Supplementary Fig. S4 and Table S2).

\section{Mechanoelectrical and morphological properties of PVDF- HFP/PEDOT NFs}

The sheet resistance of the PVDF-HFP/PEDOT mat was measured using the four-point probe method at room temperature, and the calculated value was ca. $7 \times 10^{4} \Omega$ $\mathrm{sq}^{-1}$. Figure $3 \mathrm{e}$ indicates the radius of the electrospun deposition area as a function of the tip-to-collector distance. The sheet resistance of the fiber mats was relatively constant under a variety of conditions. In addition, the optical transparency of the electrospun fibers could be 
controlled by changing the electrospinning deposition time. The optical transparencies of three samples prepared on substrates with deposition times of $0.5,1$, and 3 min are compared in Supplementary Fig. S5, which shows that both the density and effective thickness of the deposited layer increase with increasing deposition time.

Van Wyk first proposed a mechanistic deformation model for a 3D random fibrous medium under compression and demonstrated that organized 3D fiber networks with both vertically and perpendicularly grown structures were more suitable for use in filtration ${ }^{39}$. In our work, these 3D electrospun mats boasted four useful properties: (1) a high specific surface area (surface area per unit mass), (2) low solidity (or high porosity), (3) high interconnectivity of pore spaces, and (4) high sensitivity toward external pressure and mechanical durability owing to the formation of hierarchical structures. Conventional electrospun fibers have a layer-by-layer structure that consists of fibers aligned parallel to the plane of the collector. The compressive deformation of $2 \mathrm{D}$ fiber mats is relatively restricted due to the dense packing perpendicular to the collector plate. Conversely, mats with a 3D fiber network boast freedom of movement in both the parallel and perpendicular directions, enabling increased flexibility and elasticity. In a 3D conductive network, compressive stress leads to an increase in the number of contact points with a wide variation in resistance.

The 3D PVDF-HFP/PEDOT mats were subjected to a precompression treatment (up to $95 \%$ shrinkage) to improve their reliability as a pressure-sensitive material. Morphological changes in the 3D PVDF-HFP/PEDOT NF mats (ca. $1 \mathrm{~mm}$ thickness) during compressive deformation were characterized using field-emission scanning electron microscopy (FE-SEM). Changes in the thickness and resistance were also measured as a function of compression (Fig. 4a, b). The high stability of the bump core/ shell NF mats could be attributed to the spherical PEDOT nanostructures present on the PVDF-HFP fibers. The unique structures of the core/shell fibers with PEDOT bumps provide the conductive sponge mat with sufficient contact sites when it is compressed and also play a role in stress dissipation when the force is released ${ }^{40,41}$. In the pristine PVDF-HFP/PEDOT mat, several layers of fibers were randomly entangled and stacked to form a porous structure. The contact area of the fibers increased as the mat was compressed. In thousands of repeated compression cycles, the spherical bump PVDF-HFP/PEDOT 3D NF mat showed nearly no noticeable degradation, indicating that the 3D fibrillar network and spherical bump shape NFs were suitable for reusable pressure sensors; 1.4 and $2.0 \%$ reductions in the thickness were observed after 1000 and 10,000 cycles, respectively (Fig. 4c). Since the PEDOT shell was chemically deposited onto the surface of the PVDF-HFP NFs, cross-sectional morphologies of normal (flat) and spherical bump core/ shell NFs after 10,000 cycles were investigated using FIBSEM analysis. As shown in Fig. 4d and e, the PEDOT shell was uniformly coated on the surface of the PVDF-HFP NFs without peeling or wearing off of the PVDF-HFP core after the long-term cycling tests.

PVDF copolymers (e.g., PVDF-TrFE, PVDF-HFP) exhibit piezoelectric characteristics under stretching or deformation, and the application of pressure could lead to changes in both the voltage and resistance at the same time. The PVDF-HFP/PEDOT pressure sensor showed changes in both resistance and voltage under an applied pressure (Supplementary Fig. S6). Although the applied pressure led to the changes in the voltage and resistance at the same time, the two factors were not affected by each other because they had different transduction mechanisms. The sensor generated a weak open-circuit voltage signal under applied pressure, while it underwent a large change in resistance owing to the spherical bump 3D NF structure (Supplementary Fig. S7). Therefore, the high pressure sensitivity of the sensor prepared in this research was mainly caused by the change in resistance.

To demonstrate the pressure sensitivity of the 3D PVDF-HFP/PEDOT mats, the response to an applied pressure was monitored by measuring the relative change in the sensor output current, $\Delta I / I_{0}=\left(I-I_{0}\right) / I_{0}$, where $I_{0}$ is the initial current and $\Delta I$ is the change in the current under pressure loading. The electrospun, pressure-sensitive NF sensors were evaluated as a function of applied pressure on the surface of a unit device composed of PVDF-HFP/ PEDOT mats prepared under different deposition conditions. The pressure sensitivity, $S$, can be defined as the slope of the resulting curve $\left(S=\left(\Delta I / I_{0}\right) / \Delta P\right.$, where $P$ denotes the applied pressure). When the applied pressure increased from $1 \mathrm{~Pa}$ to $30 \mathrm{kPa}$, the resistance of the pressure sensor greatly increased due to the elastic deformation of the 3D fiber mat and the consequent increase in contact area between fibers. A pressure sensor with a higher sensitivity and mechanical durability was realized with a 3D fiber network with a fully grown spherical bump PEDOT shell, as shown in Supplementary Fig. S8.

In real-time sensing tests at pressures below ca. $10 \mathrm{kPa}$, the 3D electrospun NF mats exhibited higher sensitivities than the mats prepared with 2D fiber networks. As shown in Fig. 5a, the 3D mat prepared with 1-min-electrospun NFs showed a greatly enhanced sensitivity $\left(13.5 \mathrm{kPa}^{-1}\right)$ compared with those prepared using a conventional $2 \mathrm{D}$ electrospinning method $\left(5.1 \mathrm{kPa}^{-1}\right)$. In the high-pressure regime (>10 $\mathrm{kPa}$ ), the $S$ value was approximately $0.9 \mathrm{kPa}^{-1}$. In the low-pressure regime $(1-10 \mathrm{kPa})$, the sensor exhibited a linear response to pressure, implying the good reliability of the sensor for practical applications. This level of sensitivity is unmatched in previous reports (Supplementary Tables S3 and S4). Figure 5b and c shows the 

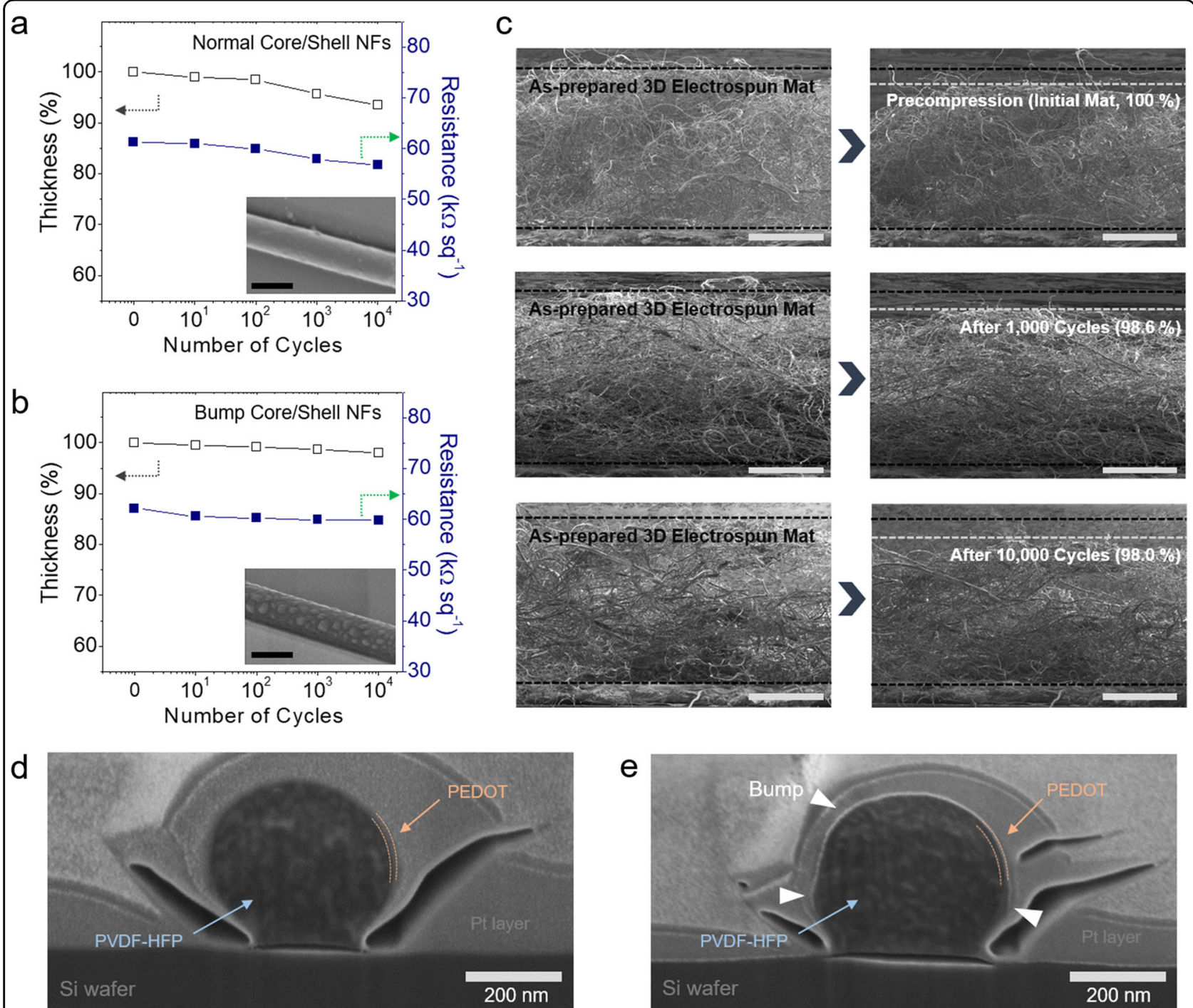

Fig. 4 Morphological changes in a 3D electrospun PVDF-HFP/PEDOT NF mat upon the application of pressure and the effects of spherical bumps on the long-term performance. a Thickness and resistance changes of a normal (flat) core/shell NF mat. After precompression treatment, the thickness of the as-prepared 3D electrospun mat was reduced to 9.9 and $9.6 \%$ for the normal core/shell NFs and the bump core/shell NFs, respectively. b Spherical bump core/shell 3D NF mats during a repeated cycling test (the inset SEM images show the morphology of a single core/ shell fiber, and the scale bar represents $500 \mathrm{~nm}$ ). c SEM images of pristine spherical bump 3D NF mats and the recovered mats after applying cyclic compressive deformation. The scale bar represents $500 \mu \mathrm{m}$. The initial height of a mat deposited for 3 min was ca. 1 mm. Cross-sectional SEM images of $\mathbf{d}$ normal (flat) and $\mathbf{e}$ spherical bump core/shell NFs after 10,000 cycles (Pt was deposited as a protecting layer during the FIB etching process)

changes in current obtained with 1-min-deposited 3D and 2D NF films, respectively, as a function of the applied voltage. The current increased with an increase in pressure from 1 to $50 \mathrm{~Pa}$, and the linear $I-V$ curves indicate Ohmic behavior with a relatively stable response to static pressure. Notably, our devices showed a high sensitivity even at voltages as low as $1 \mathrm{~V}$ with a minimum detection of $1 \mathrm{~Pa}$. Furthermore, the Brunauer-Emmett-Teller surface area of the 3D electrospun mat $\left(4.89 \mathrm{~m}^{2} \mathrm{~g}^{-1}\right)$ was larger than that of the $2 \mathrm{D}$ electrospun mat $\left(4.57 \mathrm{~m}^{2} \mathrm{~g}^{-1}\right)$, which is consistent with our results (Supplementary Fig. S9). While microstructured and nanostructured, pressure-sensitive thin films have been intensively studied, our durable, 3D, randomly nanostructured fiber mats have shown excellent sensitivity and stable performance, with rapid response $(0.4 \mathrm{~s})$ and recovery $(0.2 \mathrm{~s})$ times at applied pressures less than $10 \mathrm{kPa}^{2,13,23}$.

Repeated, real-time sensing tests were also performed. As indicated in Fig. 2, the PEDOT shell and bumps with varying thicknesses were formed by VDP. However, as shown in Supplementary Fig. S10, the manufactured spherical bump PVDF-HFP/PEDOT 3D NF sensors showed relatively small differences in the sample-tosample variation tests of 25 devices because the entangled 

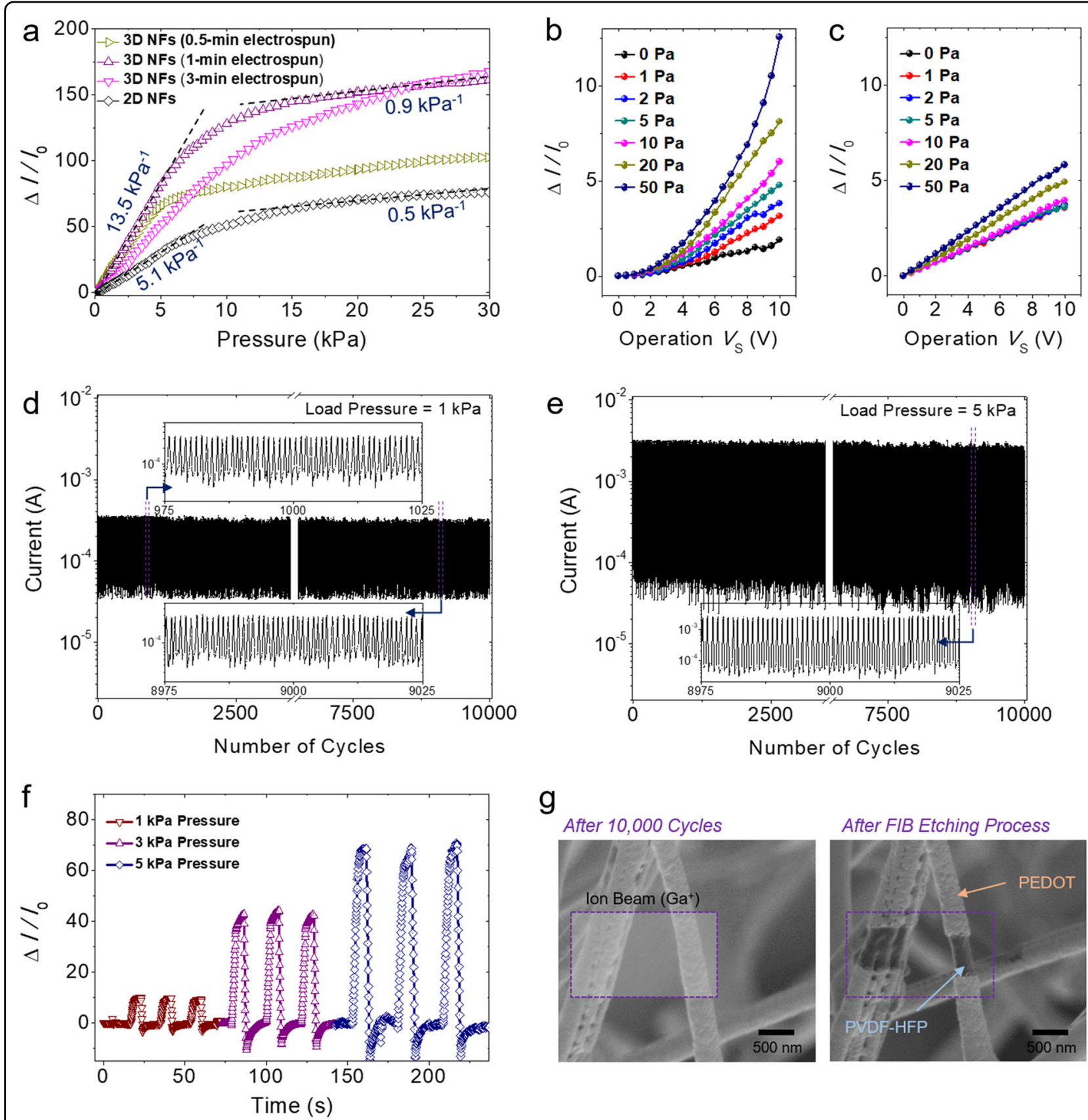

g
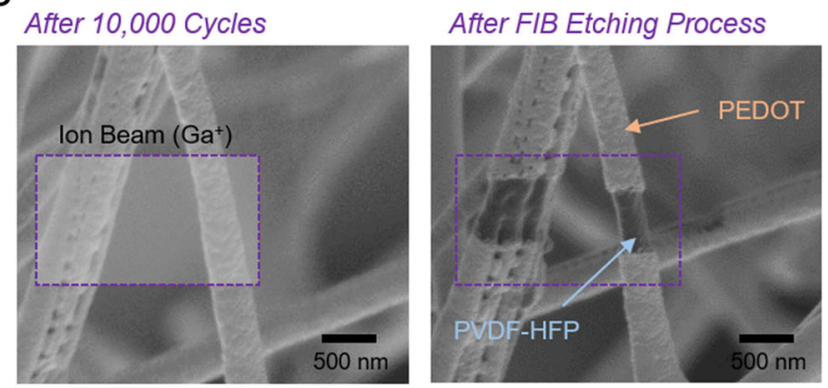

Fig. 5 Mechanoelectric properties of spherical bump PVDF-HFP/PEDOT NF sensors. a Current response $\left(\Delta l / I_{0}\right)$ vs. applied pressure plots showing the effects of 3D and 2D electrospinning on the pressure sensors. The 3D NF mat prepared by 1 min electrospinning exhibited the highest sensitivity. I-V curves of 1-min-deposited $\mathbf{b} 3 \mathrm{D}$ NF-based and $\mathbf{c} 2 \mathrm{D}$ NF-based devices under different pressure loading conditions $\left(I_{0}\right.$ is the current at $V_{s}=0 \mathrm{~V}, P=0 \mathrm{~Pa}$ ). Repeatability tests of spherical bump PVDF-HFP/PEDOT 3D NF sensors. Cyclic tests of the pressure sensor were performed over 10,000 cycles at $\mathbf{d} 1 \mathrm{kPa}$ and $\mathbf{e} 5 \mathrm{kPa}$ using the same device. $\mathbf{f}$ The real-time pressure sensing performance of spherical bump PVDF-HFP/PEDOT 3D NF sensors. $\mathbf{g}$ The morphologies of the spherical bump PVDF-HFP/PEDOT 3D NFs after 10,000 cycles measured with an FIB etching process

network is composed of thousands of fibers. As shown in Fig. $5 \mathrm{~d}$ and e, repeatability tests of the spherical bump PVDF-HFP/PEDOT 3D NF sensor were performed using the same device over 10,000 cycles at pressures of 1 and 5 $\mathrm{kPa}$. The sensor responses were relatively consistent, even up to 10,000 cycles. The developed devices maintained their response and recovery properties after multiple cycles at various pressures (Fig. 5f). The PVDF-HFP/ PEDOT pressure sensors possessed highly stable architectures without any observable structural degradation after repeated compression tests. To clarify the maintenance of the core/shell structure, the morphologies of the spherical 
bump PVDF-HFP/PEDOT 3D NFs after 10,000 cycles were investigated using an FIB etching process. As shown in Fig. 5g, the PEDOT shell remained uniform on the surface of the PVDF-HFP NFs without wearing off of the PVDF core after the long-term cycling tests.

\section{Demonstration of PVDF-HFP/PEDOT NF-based human-machine sensing platform}

Since our pressure-sensing platform is based on polymeric materials, it is well suited for the fabrication of large-scale and low-cost pressure sensor arrays. To assess the potential for applications in human-machine interfaces and E-skin for self-health monitoring, a $16 \times 10$ pressure-sensing array and a unit sensor embedded in a wearable band, respectively, were fabricated with a wireless module. Figure 6a illustrates the configuration of the sensor array matrix (left) and the unit sensor (right).

A pressure sensor array matrix is useful for various applications in which pressure mapping is required. A simple pressure-sensing matrix was prepared by forming horizontal and vertical electrodes below sensitive polymeric materials on a flexible plastic substrate (PET). Pressure mapping was performed by applying a voltage to the horizontal line and reading the resistance of each vertical line, i.e., the horizontal line was driven to a neutral voltage, and the resistance of the vertical line was monitored. In this way, all of the matrix pixels were scanned. This process was developed with our spherical bump PVDF-HFP/PEDOT-based sensors and can easily be scaled up to allow the production of large-area, flexible
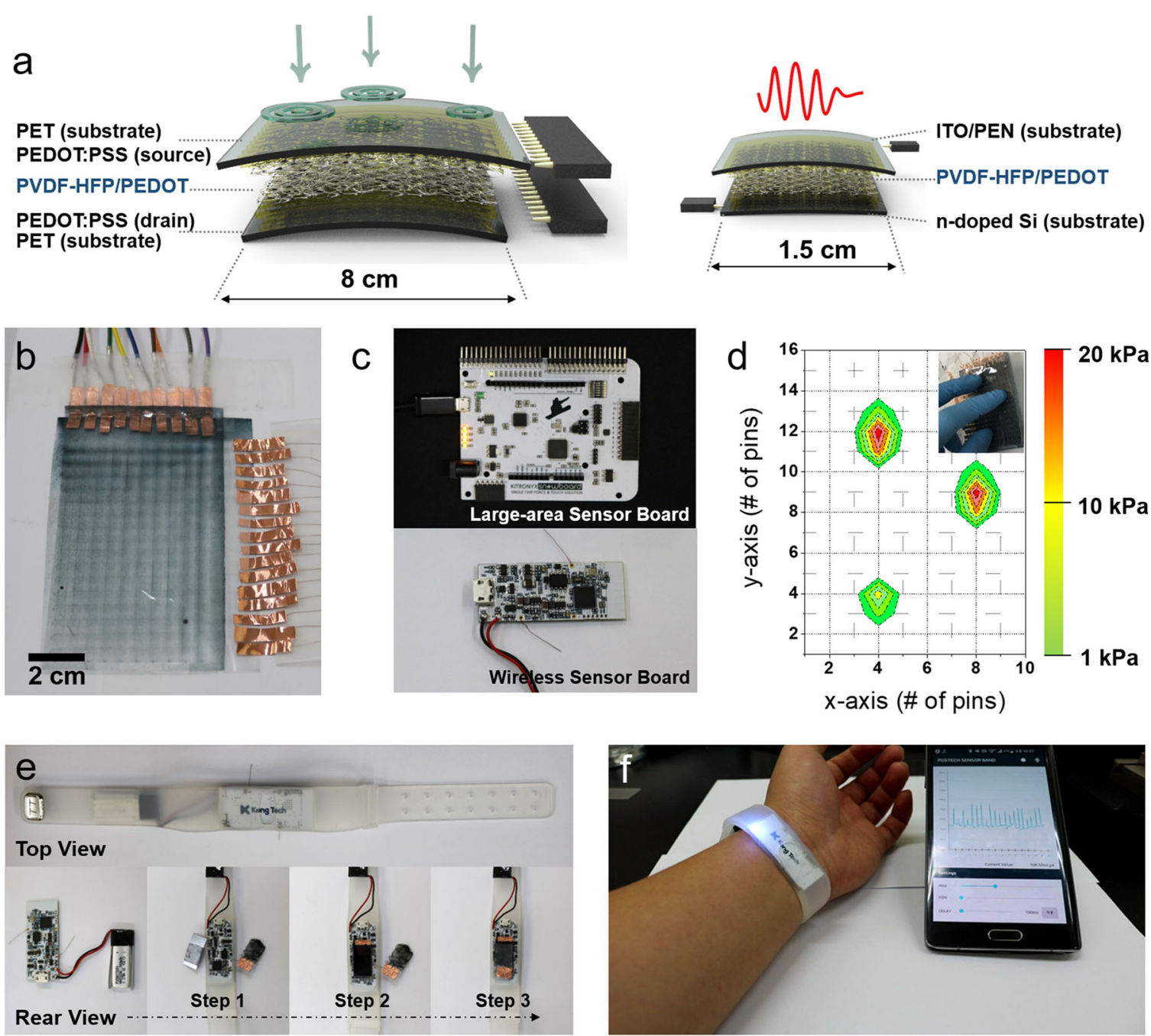

Fig. 6 Practical applications of human-machine interfaces using spherical bump PVDF-HFP/PEDOT 3D NF mats. a Schematic illustration of the sensor matrix (left) and single-unit sensor systems (right). b Optical images of a PVDF-HFP/PEDOT array on an inkjet-printed electrode PEDOT:PSS/ PET film, c a commercially available board for pressure mapping (top) and a small custom-made Bluetooth board (bottom). $\mathbf{d}$ Spatial pressure mapping of a PVDF-HFP/PEDOT array sensor. e Top and rear-view photographs of a prototype blood pressure sensor embedded in a wrist band. f Photograph of a wireless blood pressure sensor and diagnostic signals on the display of a smartphone 
pressure sensors. We created a durable 3D conductive structure and used it as a single-layer sponge-like conductive pressure-sensitive material to form a 160-pixel (in an area of $8 \times 6 \mathrm{~cm}^{2}$ ) piezoresistive pressure sensor array that can measure pressure distributions by detecting the resistance of each pixel. Figure $6 \mathrm{~b}$ shows a photograph of the $16 \times 10$ pressure sensor array consisting of a PVDFHFP/PEDOT fiber mat/PEDOT:PSS electrode/PET substrate structure fabricated using 3D electrospinning and VDP methods (Supplementary Figs. S11, S12). In addition, whole pieces of the as-prepared PVDF-HFP/PEDOTbased sensors were connected to a wireless board using copper cables and tape. The intensity of the current variation was recorded and plotted as a $2 \mathrm{D}$ pressure distribution. A commercially available sensing board with a $\mathrm{mA}$ current read (operating voltage: $5 \mathrm{~V}$ ) and a custommade Bluetooth module were used (Fig. 6c). Simultaneous finger touches at three locations on the surface of the sensor were used to test the response of the sensor to multiple external pressure points. The output current variation from each pixel was recorded and is plotted in Fig. $6 \mathrm{~d}$, which shows the spatial distribution of the pressure via color contrast mapping. The measured spatial distribution of pressure was highly consistent with the location of each finger touch. The pixel resolution was approximately $2 \times 2 \mathrm{~mm}^{2}$ and was limited by the size of the PEDOT:PSS electrodes and the copper tape used in the device (Supplementary Movie S1).

A wearable band containing a single-unit pressure sensor was fabricated to monitor blood pressure in real time. PVDF-HFP fibers were collected directly on a flexible ITO-coated PEN substrate and an $n$-doped Si wafer. The pressure sensor device was composed of two electrodes on both sides of a spherical bump PVDF-HFP/ PEDOT mat composite (see the right image in Fig. $6 \mathrm{a}$ and Supplementary Fig. S13), which had a single output and a small, sandwich-like structure. Top-view and rear-view photographs of a wireless blood pressure sensor band are presented in Fig. 6e. Real-time sensing experiments of human blood pressure yielded well-resolved signals on a smartphone screen through a custom-made user interface (Fig. 6f and Supplementary Movie S2).

\section{Conclusion}

In summary, conductive core/shell polymer NFs were fabricated using 3D electrospinning and VDP methods. The resulting material exhibited the mechanoelectrical characteristics of pressure-sensitive PVDF-HFP/PEDOT $3 \mathrm{D}$ electrospun NF mats. By applying a novel electrospinning technique with repulsive forces that enable 3D fiber stacking, we successfully fabricated a conductive, sponge-like polymeric material composed of PVDF-HFP/ PEDOT with a thickness of approximately $1 \mathrm{~mm}$. The sponge-like material exhibited a pressure sensitivity of
$13.5 \mathrm{kPa}^{-1}$, which is more than twice that of conventional electrospun mats $\left(5.1 \mathrm{kPa}^{-1}\right)$. The fabricated fibers were coated with a hierarchy of conductive nanostructures consisting of spherical bumps. The sensor response yielded a minimum detection of $1 \mathrm{~Pa}$ and was resilient even up to 10,000 compressive cycles. Furthermore, we proposed a facile means of fabricating practical pressure sensors by constructing IoT platforms. This concept was demonstrated with a highly sensitive, large-area pressure mapping sensor (160 pixels in an area of $8 \mathrm{~cm} \times 6 \mathrm{~cm}$ ), and a wireless blood pressure monitoring band. The developed method represents a new route toward the polymer-based fabrication of high-performance, pressure-sensitive materials with high mechanical durability and operational stability. In addition, the pressure-sensing platform described herein is suitable for a wide range of applications in biomedical diagnostics and environmental monitoring and is a promising candidate for the fabrication of lowcost artificial E-skin, flexible circuits, and wearable electronics.

\section{Acknowledgements}

This work was supported by a National Research Foundation of Korea (NRF) grant (No. 2017R1E1A1A01074090), the Nano Material Technology Development Program (No. 2017M3A7B8063825), and the Center for Advanced Soft Electronics under the Global Frontier Research Program (No. 2013M3A6A5073175) funded by the Ministry of Science and ICT (MSIT), Korea. This work was also supported by the Green Science Program of POSCO company, Korea.

Conflict of interest

The authors declare that they have no conflict of interest.

Publisher's note

Springer Nature remains neutral with regard to jurisdictional claims in published maps and institutional affiliations.

Supplementary information is available for this paper at https:/doi.org/ 10.1038/s41427-018-0041-6.

Received: 16 November 2017 Revised: 16 March 2018 Accepted: 24 March 2018.

Published online: 18 June 2018

\section{References}

1. Gao, W. et al. A fully integrated wearable sensor arrays for multiplexed in situ perspiration analysis. Nature 529, 509-514 (2016).

2. Tee, B. C.-K. et al. A skin-inspired organic digital mechanoreceptor. Science $\mathbf{3 5 0 ,}$ 313-316 (2015).

3. Kaltenbrunner, M. et al. An ultra-lightweight design for imperceptible plastic electronics. Nature 499, 458-463 (2013).

4. Feiner, R. et al. Engineered hybrid cardiac patches with multifunctional electronics for online monitoring and regulation of tissue function. Nat. Mater. 15, 679-685 (2016).

5. Dai, X., Zhou, W., Gao, T., Liu, J. \& Lieber, C. M. Three-dimensional mapping and regulation of action potential propagation in nanoelectronics-innervated tissues. Nat. Nanotechnol. 11, 776-782 (2016).

6. Anikeeva, P. \& Koppes, R. A. Restoring the sense of touch. Science $\mathbf{3 5 0}$ 274-275 (2015).

7. Tan, D. W. et al. A neural interface provides long-term stable natural touch perception. Sci. Transl. Med. 6, 257ra138 (2014). 
8. Chortos, A., Liu, J. \& Bao, Z. Pursuing prosthetic electronic skin. Nat. Mater. 15, 937-950 (2016)

9. Lee, $\mathrm{H}$. et al. A graphene-based electrochemical device with thermoresponsive microneedles for diabetes monitoring and therapy. Nat. Nanotechnol. 11, 566-572 (2016).

10. Zang, Y., Zhang, F., Di, C.-a \& Zhu, D. Advances of flexible pressure sensors toward artificial intelligence and health care applications. Mater. Horiz. 2, 140-156 (2015).

11. $\mathrm{Ha}, \mathrm{M}$. et al. Bioinspired interlocked and hierarchical design of $\mathrm{ZnO}$ nanowire arrays for static and dynamic pressure-sensitive electronic skins. Adv. Funct. Mater. 25, 2841-2849 (2015).

12. Choong, C. L. et al. Highly stretchable resistive pressure sensors using a conductive elastomeric composite on a micropyramid array. Adv. Mater. 26, 3451-3458 (2014).

13. Lee, J. et al. Conductive fiber-based ultrasensitive textile pressure sensor for wearable electronics. Adv. Mater. 27, 2433-2439 (2015).

14. Lee, J. S., Shin, K. Y., Cheong, O. J., Kim, J. H. \& Jang, J. Highly sensitive and multifunctional tactile sensor using free-standing ZnO/PVDF thin film with graphene electrodes for pressure and temperature monitoring. Sci. Rep. $\mathbf{5}$, 7887 (2015).

15. Kim, D. I. et al. A sensor array using multi-functional field-effect transistors with ultrahigh sensitivity and precision for bio-monitoring. Sci. Rep. 5, 12705 (2015)

16. Zhu, B. et al. Skin-inspired haptic memory arrays with an electrically reconfigurable architecture. Adv. Mater. 28, 1559-1566 (2016).

17. Larson, C. et al. Highly stretchable electroluminescent skin for optical signaling and tactile sensing. Science 351, 1071-1074 (2016).

18. Lee, T., Lee, W., Kim, S.-W., Kim, J. J. \& Kim, B.-S. Flexible textile strain wireless sensor functionalized with hybrid carbon nanomaterials supported $\mathrm{ZnO}$ nanowires with controlled aspect ratio. Adv. Funct. Mater. 26, 6206-6214 (2016).

19. Bae, G. Y. et al. Linearly and highly pressure-sensitive electronic skin based on a bioinspired hierarchical structural array. Adv. Mater. 28, 5300-5306 (2016).

20. Lou, Z., Chen, S., Wang, L., Jiang, K. \& Shen, G. An ultra-sensitive and rapid response speed graphene pressure sensors for electronic skin and health monitoring. Nano Energy 23, 7-14 (2016).

21. Tian, $\mathbf{H}$. et al. A graphene-based resistive pressure sensor with record-high sensitivity in a wide pressure range. Sci. Rep. 5, 8603 (2015).

22. Garain, S., Jana, S., Sinha, T. K. \& Mandal, D. Design of in situ poled $\mathrm{Ce}(3$ + )-doped electrospun PVDF/graphene composite nanofibers for fabrication of nanopressure sensor and ultrasensitive acoustic nanogenerator. ACS Appl. Mater. Interfaces 8, 4532-4540 (2016).

23. Lee, S. et al. A transparent bending-insensitive pressure sensor. Nat. Nanotechnol. 11, 472-478 (2016).

24. Merlini, C., Barra, G. M. O., Medeiros, A. T. \& Pegoretti, A. Electrically pressure sensitive poly(vinylidene fluoride)/polypyrrole electrospun mats. RSC Adv. 4, 15749 (2014).
25. Tiwari, M. K., Yarin, A. L. \& Megaridis, C. M. Electrospun fibrous nanocomposites as permeable, flexible strain sensors. J. Appl. Phys. 103, 044305 (2008).

26. Ghosh, S. K. et al. Electrospun gelatin nanofiber based self-powered bio-e-skin for health care monitoring. Nano Energy 36, 166-175 (2017).

27. Zhou, Y. M. et al. Highly sensitive, self-powered and wearable electronic skin based on pressure-sensitive nanofiber woven fabric sensor. Sci. Rep. 7, 12949 (2017).

28. Hwang, T. H., Lee, Y. M., Kong, B. S., Seo, J. S. \& Choi, J. W. Electrospun core-shell fibers for robust silicon nanoparticle-based lithium ion battery anodes. Nano Lett. 12, 802-807 (2012).

29. Persano, L. et al. High performance piezoelectric devices based on aligned arrays of nanofibers of poly(vinylidenefluoride-co-trifluoroethylene). Nat. Commun. 4, 1633 (2013).

30. Yao, H. B. et al. A flexible and highly pressure-sensitive graphene-polyurethane sponge based on fractured microstructure design. Adv. Mater. 25, 6692-6698 (2013).

31. Bonino, C. A. et al. Three-dimensional electrospun alginate nanofiber mats via tailored charge repulsions. Small 8, 1928-1936 (2012).

32. Cai, S., Xu, H., Jiang, Q. \& Yang, Y. Novel 3D electrospun scaffolds with fibers oriented randomly and evenly in three dimensions to closely mimic the unique architectures of extracellular matrices in soft tissues: fabrication and mechanism study. Langmuir 29, 2311-2318 (2013).

33. Chun, S., Hong, A., Choi, Y., Ha, C. \& Park, W. A tactile sensor using a conductive graphene-sponge composite. Nanoscale 8, 9185-9192 (2016).

34. $\mathrm{Xu}$, J. et al. A hierarchical carbon derived from sponge-templated activation of graphene oxide for high-performance supercapacitor electrodes. Adv. Mater. 28, 5222-5228 (2016).

35. Wang, R. et al. The fabrication and tribological behavior of epoxy composites modified by the three-dimensional polyurethane sponge reinforced with dopamine functionalized carbon nanotubes. Appl. Surf. Sci. 360, 37-44 (2016).

36. Wang, S. et al. Stretchable polyurethane sponge scaffold strengthened shear stiffening polymer and its enhanced safeguarding performance. ACS Appl. Mater. Interfaces 8, 4946-4954 (2016).

37. Jang, J. \& Lim, B. Facile fabrication of inorganic-polymer core-shell nanostructures by a one-step vapor deposition polymerization. Angew. Chem. Int. Ed. Engl. 42, 5600-5603 (2003).

38. Sun, B. et al. Self-assembly of a three-dimensional fibrous polymer sponge by electrospinning. Nanoscale 4, 2134-2137 (2012).

39. van Wyk, C. M. Note on the compressibility of wool. J. Text. Inst. Trans. 37, T285-T292 (1946)

40. Jian, M. Q. et al. Flexible and highly sensitive pressure sensors based on bionic hierarchical structures. Adv. Funct. Mater. 27, 1606066 (2017).

41. Park, J. et al. Fingertip skin-inspired microstructured ferroelectric skins discriminate static/dynamic pressure and temperature stimuli. Sci. Adv. 1, e1500661 (2015). 